\title{
CRISPR-based editing reveals edge-specific effects in biological networks
}

Yi Li $^{1,2}$, Chance M. Nowak ${ }^{2,3}$, Daniel Withers ${ }^{1,2}$, Alexander Pertsemlidis ${ }^{4}$, Leonidas Bleris ${ }^{1,2,3 *}$

${ }^{1}$ Department of Bioengineering

The University of Texas at Dallas, 800 West Campbell Road, Richardson TX 75080 USA

${ }^{2}$ Center for Systems Biology

The University of Texas at Dallas, 800 West Campbell Road, Richardson TX 75080 USA

${ }^{3}$ Department of Biological Sciences

The University of Texas at Dallas, 800 West Campbell Road, Richardson TX 75080 USA

${ }^{4}$ Greehey Children's Cancer Research Institute

The University of Texas Health Science Center at San Antonio, 8403 Floyd Curl Drive, San Antonio TX 78229 USA

* To whom correspondence should be addressed: Leonidas Bleris, The University of Texas at Dallas, NSERL 4.708, 800 West Campbell Road, Richardson TX 75080 USA

Email: bleris@utdallas.edu

Phone: 972-883-5785 


\begin{abstract}
Unraveling the properties of biological networks is central to understanding normal and disease cellular phenotypes. Networks consist of functional elements (nodes) that form a variety of diverse connections (edges) with each node being a hub for multiple edges. Herein, in contrast to node-centric network perturbation and analysis approaches, we present a high-throughput CRISPR-based methodology for delineating the role of network edges. Ablation of network edges using a library targeting 93 miRNA target sites in 71 genes reveals numerous edges that control, with variable importance, cellular survival under stress. To compare the impact of removing nodes versus edges in a biological network, we dissect a specific p53-microRNA pathway. In summary, we demonstrate that network edges are critical to the function and stability of biological networks. Our results introduce a novel genetic screening opportunity via edge ablation and highlight a new dimension in biological network analysis.
\end{abstract}




\section{Introduction}

We focus on the network formed by p53 and its upstream and downstream regulators, which is critical to cell health, yet incompletely understood. Since its discovery in 1979 (1), p53 has been shown to play a crucial role in maintaining genomic stability (2), with more than $50 \%$ of human cancers harboring mutant or deleted p53 (3). Under normal conditions, the p53 protein exists in a latent form and at low concentration, but in response to various cellular stress signals such as DNA damage, hypoxia, and oncogene expression, posttranslational modification of p53 results in its stabilization and accumulation (4). As most human malignancies shut down the p53 tumor-suppressing responses, p53 is one of the most promising targets for drug interventions in cancer therapy.

A class of post-transcriptional regulators, called microRNAs (miRNAs), is directly associated with p53, either regulating mRNA responsible for p53 production or being regulated by p53 and its partners $(5,6)$. miRNAs, in their mature forms, are small non-coding RNAs approximately 22 nucleotides in length that act as major regulators of gene expression. Since miRNAs are involved in critical cellular processes such as growth, differentiation, and apoptosis, the loss of critical miRNAs in a given cell type can have significant implications for cell fate (7-9). Studies have revealed extensive crosstalk between the p53 network and miRNAs, but the specifics of how miRNAs participate in the regulation of p53 signaling and what they contribute to the role of p53 as a tumor suppressor remain largely elusive.

In miRNA-based networks the edges are regulatory interactions between miRNAs and target mRNAs. These interactions are mediated by sequence complementarity and therefore are susceptible to genetic variation occurring in either the miRNA or the target site. Variation in miRNA binding sites has been associated with numerous diseases, including Tourette Syndrome (10), rheumatoid arthritis (11), lupus (12), psoriasis (13), Crohn's disease $(14,15)$, Parkinson's disease (16), hypertension $(17,18)$, diabetes and obesity $(19,20)$, and multiple cancers (21-25). In the context of p53 signaling (26), miR-34a regulates HDM4, a strong repressor of 
p53, creating a positive feedback loop in which high levels of miR-34a de-repress p53 which in turn transcriptionally up-regulates the expression of miR-34a.

Network edges (e.g. miRNA-gene target interactions) are therefore central to the function and stability of biological pathways, yet comprehensive studies focusing on edges remain few. Today, we have the unprecedented opportunity to dissect individual cells and pathways with single-nucleotide specificity using genome editing. The most widely-adopted editing methodology to date is the bacterial type II clustered regularly interspaced short palindromic repeats (CRISPR) system consisting of the CRISPR-associated protein Cas9 derived from Streptococcus pyogenes (SpCas9), a DNA endonuclease, and a guide RNA, which directs the binding of Cas9 to a DNA target upstream of a protospacer adjacent motif (PAM).

The CRISPR technology has revolutionized our ability to probe and edit the human genome in vitro and in vivo, including targeted gene disruption, insertion, single-nucleotide mutation, and chromosomal rearrangements (27-29). Furthermore, pooled sgRNA libraries can be used for versatile in vitro screening to investigate phenotypes of interest. Recent examples include screens identifying genes conferring drug resistance (30), genes involved in metastasis (31), and long non-coding RNAs (lncRNAs) regulating human cancer cell growth (32). Thus far, pooled sgRNA libraries have been applied to transcribed loci, which correspond to network nodes. Here, we selectively remove edges in the miRNA-p53 network, using a first-of-a-kind CRISPRbased screen. We demonstrate that removing edges sheds new light on pathways, in ways not achievable through node-based approaches, which may lead to novel and non-obvious therapeutic opportunities.

\section{Results}

\section{CRISPR-based screen for microRNA target editing}

We focus on five miRNAs - miR-34a, miR-145, miR-192, miR-194 and miR-215 - which are known to be directly or indirectly regulated by p53, and play elaborate roles in the p53 pathway $((33,34)$, review (35)). The target genes for each miRNA were compiled from miRTarBase (36). We selected targets that have been experimentally validated by multiple methods, including luciferase reporter assay, western blot, and quantitative 
RT-PCR (qRT-PCR) (Supplementary Table 1). For each of the target genes, the miRNA target sites within its

3'UTR were determined using TargetScan (37). In total, 93 miRNA target sites were identified across the 71 target genes. The miRNAs and the 71 target genes are the nodes of the derived network, while the experimentally verified and high-confidence predicted interactions between the nodes, including interactions between miRNAs and target genes and between target genes themselves, are the network edges (Figure 1a). Since SpCas9-mediated NHEJ typically introduces short insertions or deletions (indels) near its cutting site, we designed sgRNAs in which a PAM is adjacent to the miRNA target seed sequences within the 3'UTR (38) (Supplementary Table 1).

Next, we constructed a pooled CRISPR sgRNA library, containing both SpCas9 and sgRNA expression cassettes (39). Equimolar amounts of the 93 pairs of sgRNA oligonucleotides were mixed and cloned into a lentiviral vector (lentiCRISPRv2). The resulting plasmid library was subjected to sequencing, to confirm library complexity. The resulting reads displayed a 20-bp "noisy" sgRNA target sequence matching the expected pattern from the sgRNA mixture (Supplementary Figure 1). Subsequently, the lentiviral library was used to infect HCT116 wild-type (WT) and HCT116 p53/- cells at a multiplicity of infection (MOI) of 0.3 , which has been shown (40) to yield at most one integration of the sgRNA cassette in the majority of cells (Figure 1b). To verify the complexity of our resulting libraries in cells (named LIB-WT and LIB-p53 ${ }^{-/-}$) was maintained, the sgRNA locations were amplified from genomic DNA and subjected to Sanger sequencing, which again displayed the expected pattern (Supplementary Figure 2).

In parallel, to test the efficacy of the viral system, we prepared two CRISPR lentiviral vectors that target the open reading frame (ORF) of the zeocin resistance gene (target 1: 5'-TCGCCGGAGCGGTCGAGTTCTGG; target 2: 5'-CTCACCGCGCGCGACGTCGC-CGG; PAM underlined), and stably integrated them into cell line Flp-In-293 which harbors the zeocin resistance gene (ThemoFisher Scientific). As shown in Supplementary Figure 3, disruption of the Zeocin resistance gene abolished resistance to Zeocin $(100 \mu \mathrm{g} / \mathrm{mL})$ in the two resulting cell lines (FLP-EDIT1 and FLP-EDIT2), compared to the parental Flp-In-293 cells. 
Using the established cell lines (LIB-WT and LIB-p53 ${ }^{-/}$), we focused on the role of miR-34a in the overall p53-miRNA network (Figure 1a). miR-34a is transcriptionally activated by p53 and induces an antiproliferative phenotype including senescence, cell cycle arrest at the G1 phase, and apoptosis $(41,42)$. In turn, overexpression of miR-34a increases p53 protein level and stability (33). Importantly, our established cell lines (LIB-WT and LIB-p53 ${ }^{-/-}$) have low-level baseline miR-34a expression (Supplementary Figure 5) while the ectopic miR-34a delivery results, on average, in a 71-fold increase.

We adopted a survival assay mediated by ectopic exposure to miR-34a mimics. Specifically, we treated both cell lines (LIB-WT and LIB-p53-- ) to $25 \mathrm{nM}$ of miR-34a mimic for 6 days. Cells were harvested at day 0 (before miRNA treatment) and at day 6. For each sample, sgRNA constructs were amplified from genomic DNA and subjected to the Illumina NGS Amplicon Sequencing to assess the relative abundance for each of the 93 sgRNA target sequences (Supplementary Table 2). The most enriched or depleted sgRNA targets, defined by fold-changes between Day 6 and Day 0 larger than 10, were identified for both LIB-WT and LIB-p53 $3^{-/-}$cells (Figure 2, Supplementary Table 3).

Intriguingly, RBX1 (RING-box protein 1), a RING subunit of SCF (Skp1, Cullins, F-box) E3 ubiquitin ligases, was highly enriched in both cell lines. Although not a direct target of miR-34a, overexpression of RBX1 has been demonstrated to increase cancer cell survival (43), and thus could serve as a general response mechanism to cellular stress induced by ectopic miR-34a. Additionally, for a subset of gene targets, we observed differential response to miR-34a treatment between the LIB-WT and LIB-p53 ${ }^{-/-}$cells (Supplementary Table 3). For example, the sgRNA targeting the anti-apoptotic gene Bcl-2 was enriched in the LIB-p53 ${ }^{-/-}$cells after miR-34a treatment while no enrichment was observed in the LIB-WT cells (Supplementary Figure 4).

\section{Node perturbations versus edge edits in biological networks}

Our edge editing approach revealed (Figure 2b) several clones that are enriched or depleted after prolonged exposure to ectopic miR-34a. To assess the impact of edge removal (through ablation of 
miRNA:target interactions) we focused on $\mathrm{Bcl}-2$, a gene that in response to miR-34a has different response between the two cell lines (Figure 2b) and is known to be involved in cell survival (44).

Returning to the HCT116 wild-type (WT) and HCT116 p53 ${ }^{-/-}$cells, we removed the miR-34a target site from the Bcl-2 locus. We prepared a single sgRNA construct designed against the Bcl2 3'UTR and established stable cell lines (BCL2tgt-WT and BCL2tgt-p53 $3^{-/}$) using the same viral delivery system. Sanger sequencing of PCR products spanning the sgRNA target site showed that edits (indels) occurred immediately upstream of the PAM (Supplementary Figure 6, AGG, highlighted) in both cell lines.

Treating delivery of ectopic miR-34a mimic as perturbation of a network node and removal of the miR34a/Bcl-2 interaction as perturbation of a network edge (Figure 3a), there are four possible combinations (node and edge present/absent). When mir-34a levels are low (i.e. the node is absent) the presence or absence of the edge does not impact survival (Supplementary Figure 7) of either cell line.

In the context of node perturbation, the introduction of ectopic miR-34a in wild-type cells induces apoptosis (Figure 3b right panel and Supplementary Figure 8; cell viability is $87.6 \%$ without the miR-34a node, and $71.6 \%$ with the miR-34a node, with a p-value of 0.006$)$. Similar changes were observed in p53 $3^{-/-}$cells (Figure 3b left panel and Supplementary Figure 8; cell viability is $86.1 \%$ without the miR-34a node, and $80.0 \%$ with the miR-34a node, with a p-value of 0.002). In this case, perturbing the miR-34a node results in the same behavior for both $\mathrm{p} 53$ wild-type and $\mathrm{p} 53^{-/-}$cells.

In the context of edge perturbation, we observe that the response of the two cell lines to ectopic miR-34a is sensitive to the presence of the miR-34a/Bcl-2 edge. Specifically, removing the ability of miR-34a to regulate Bcl-2 in the p53-- cells induces apoptosis (Figure 3b left panel and Supplementary Figure 8; cell viability is $84.8 \%$ without the miR-34a/Bcl-2 edge, and $80.0 \%$ with the miR-34a/Bcl-2 edge, with a p-value of 0.015$)$, while no such phenotypic changes are observed in p53 WT cells (Figure 3b right panel and Supplementary Figure 8; cell viability is $72.0 \%$ without the miR-34a/Bcl-2 edge, and $71.6 \%$ with the miR-34a/Bcl-2 edge, with 
a p-value of 0.90$)$. We note that the same conclusions can be drawn when quantifying the early- or lateapoptotic cells (Supplementary Figure 9).

To further explore the response to miR-34a in BCL2tgt-WT cells, we quantified expression of the Bcl-2 mRNA in response to miR-34a treatment using qRT-PCR. As expected, miR-34a suppresses the expression of Bcl-2 mRNA in the WT and $\mathrm{p} 53^{-/-}$cells by $55 \%$ and $40 \%$, respectively (Supplementary Figure 10). In the BCL2tgt-p53 ${ }^{-/}$cells, ectopic miR-34a has a minimal effect on Bcl-2 mRNA level (95\% compared to the control-treated sample, with a p-value of 0.71). In the BCL2tgt-WT cells, ectopic miR-34a results in a significant down-regulation of Bcl-2 expression (62\% compared to the control-treated sample, with a p-value of 0.028), possibly due to additional $\mathrm{p} 53$-miR-34a regulatory mechanisms.

\section{Discussion}

Biological networks consist of nodes and the interactions between them (i.e. edges). Conventional screening methods remove one node at a time, disrupting all edges connected to that node, and therefore producing a relatively blunt effect. An inhibitor that perturbs or removes a single node yields diverse and systemic changes in the whole network through both direct and indirect connections (45), which may explain the heterogeneity observed with single-molecule associated therapeutics.

Our approach reveals edge-specific effects related to the pro-apoptotic p53 and anti-apoptotic Bcl-2 proteins, focal nodes of apoptotic signaling. Normally, p53-dependent inhibition of Bcl-2 and induction of BAX, PUMA and NOXA overcome the anti-apoptotic threshold set by Bcl-2 family members. Conceivably, the difference in apoptosis observed in the BCL2tgt-WT and BCL2tgt-p53 ${ }^{-/}$cells under miR-34a treatment (Figure 3b) may be explained by the presence of wild type p53-dependent upregulation of PUMA or NOXA in p53 WT cells and not in p53 ${ }^{-/-}$cells. Additionally, p53 could disrupt the binding of POU4F1 to the promoter of Bcl-2 and thus indirectly down-regulate Bcl-2 expression (Supplementary Figure 10).

Taken together, our results show that the disruption of the edge between miR-34a and Bcl-2 can revert the miR-34a triggered apoptotic effects in a p53-deficient cell model. In WT cells, introducing miR-34a 
presumably indirectly triggers the suppression of the expression of Bcl-2 via an alternative p53-related pathway, which subsequently leads to increased apoptosis.

In conclusion, our CRISPR-mediated edge screening can be used to dissect critical biological interactions essential to cell survival. More generally, we demonstrate that the subtle effect of our edge removal methodology offers superior resolution and granularity in the analysis of biological networks and can lead to the identification of previously hidden interactions and opportunities for intervention. 


\section{Materials and Methods}

\section{Preparation of the CRISPR plasmid library}

The CRISPR plasmid library was prepared by following the lentiCRISPRv2 cloning protocol provided by Dr. Feng Zhang (Department of Biology, MIT). Briefly, for each identified sgRNA target (20 nt), two oligos were synthesized. The first oligo was designed as 5'-CACCG-(20 nt sgRNA target sequence)-3'. The second oligo was designed as 5'-AAAC-(20 nt reverse complement of the sgRNA target sequence)-C-3'. All 93 pairs of oligonucleotides were synthesized by Sigma-Aldrich using its customized 96-well plate format (Supplementary Table 4). Each well contained the pair of oligos for a specific sgRNA target (100 nmol for each). The oligo pairs were reconstituted using $100 \mu \mathrm{L}$ of $\mathrm{dH}_{2} \mathrm{O}$. For annealing the oligo pairs, $2 \mu \mathrm{L}$ of each of the reconstituted oligo solutions was mixed with $2 \mu \mathrm{L}$ of 10X T4 DNA Ligase Buffer (New England Biolabs,

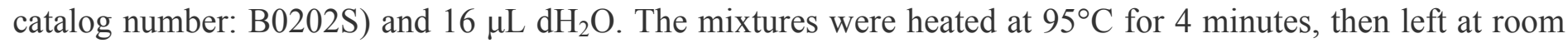
temperature for 60 minutes. $1 \mu \mathrm{g}$ of the lentiCRISPRv2 plasmid (Addgene, catalog number: 52961) was digested with $1 \mu \mathrm{L}$ Esp3I (ThermoFisher Scientific, catalog number: ER0451) at $37^{\circ} \mathrm{C}$ for 1 hour and run out on an X\% agarose gel. The $12 \mathrm{~kb}$ band was extracted using QIAquick Gel Extraction Kit (Qiagen, catalog number:

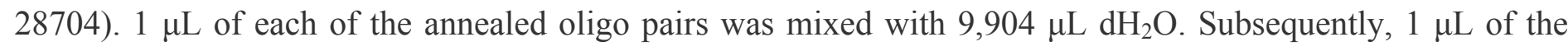
oligo mixture was ligated with Esp3I-digested lentiCRISPRv2 using T4 DNA Ligase (New England Biolabs, catalog number: M0202S). To ensure the integrity of library complexity, XL10-Gold Ultracompetent cells (Agilent, catalog number: 200314) were transformed, and more than 300 individual clones were pooled together to prepare the CRISPR plasmid library. To confirm library complexity, the plasmid library was subjected to Sanger sequencing (Genewiz) using primer P1 and analyzed using FinchTV (Geospiza).

\section{Generation of the CRISPR lentiviral screen library}

To generate the lentiviral vectors, HEK293T cells were grown to 50-70\% confluence and then transfected with $3.3 \mu \mathrm{g}$ of the CRISPR plasmid library, $3.3 \mu \mathrm{g}$ of the pMD2-VSVG plasmid, and $3.3 \mu \mathrm{g}$ of the psPAX2 plasmid using $20 \mathrm{~mL}$ of JetPRIME (Polyplus, catalog number: 114-01). $24 \mathrm{~h}$ later, the medium was removed and 
replenished with $5 \mathrm{~mL}$ of complete growth medium. In the next 3 days, the growth medium containing lentiviral

vectors was harvested, and $5 \mathrm{~mL}$ of fresh complete growth medium was replenished. The final pooled $15 \mathrm{~mL}$ growth medium was centrifuged at 3,000 rpm for $15 \mathrm{~min}$ at $4^{\circ} \mathrm{C}$ to remove cell debris. The supernatant was filtered through a $0.45 \mu \mathrm{m}$ filter, dispensed into $1-2 \mathrm{~mL}$ aliquots and stored at $-80^{\circ} \mathrm{C}$. Viral titers were determined using qPCR Lentivirus Titration Kit (ABMGood, catalog number: LV900) following manufacturer's instructions. Briefly, $2 \mu \mathrm{L}$ of viral stock was mixed with $18 \mu \mathrm{L}$ of Virus Lysis Buffer and incubated at room temperature for 3 minutes. This viral lysate, together with positive control (STD1), positive control (STD2), and negative control (NTC), were subjected to qRT-PCR. Finally, the titer of the viral stock was calculated based on the formula provided by the manufacturer and determined to be $2.07 \times 10^{7} \mathrm{IU} / \mathrm{mL}$. To generate the LIB-WT and LIB-p53-/- stable cells, $\sim 10$ million cells were seeded onto a $10 \mathrm{~cm}$ petri dish. 16 hours later, cells were transduced using the lentiviral vectors at a multiplicity of infection (MOI) of 0.3 . 48 hours post-transduction, cells were treated with $0.5 \mu \mathrm{g} / \mathrm{mL}$ of puromycin (ThermoFisher Scientific, catalog number: A1113802). Polyclonal stable cell line libraries were established after $\sim 2$ weeks of drug selection.

\section{Sanger amplicon sequencing}

To confirm the complexity of the LIB-WT and LIB-p53-- cell line libraries, total genomic DNA was isolated from LIB-WT and LIB-p53/-- cells using the DNeasy Blood \& Tissue Kit (Qiagen, catalog number: 69504). The cDNA fragments harboring the sgRNA target sequences were PCR amplified by using 100 ng of the genomic DNA and primers $\mathrm{P} 2$ and P3. PCR conditions were one cycle of 30 seconds at $98^{\circ} \mathrm{C}, 40$ cycles of 10 seconds at $98^{\circ} \mathrm{C}, 30$ seconds at $60^{\circ} \mathrm{C}$, and 30 seconds at $72^{\circ} \mathrm{C}$. The $181 \mathrm{bp}$ product was then subjected to direct Sanger sequencing using primer P2 and analyzed using FinchTV (Geospiza). To determine editing efficiency, total genomic DNA was isolated from BCL2tgt-WT and BCL2tgt-p53-- cells using the DNeasy Blood \& Tissue Kit (Qiagen, catalog number: 69504). cDNA fragments harboring the miR-34a target site within the 3'UTR of BCL2 were PCR amplified by using 100 ng of genomic DNA and primers P8 and P9. The 191 bp product was then subjected to direct Sanger sequencing using primer P9 and analyzed using FinchTV (Geospiza). 


\section{Next generation sequencing}

To determine the relative abundance of the 93 sgRNA target sequences before and after the CRISPR screen, total genomic DNA was isolated from miR-34a-treated LIB-WT and LIB-p53 $3^{-/-}$cells at days 0 and 6 using the DNeasy Blood \& Tissue Kit (Qiagen, catalog number: 69504). cDNA fragments harboring the sgRNA target sequences were PCR amplified by using 100 ng of the genomic DNA and primers P10 and P11, which added the 5'-overhang adapter sequence (5'-TCGTCGGCAGCGTCAGATGTGTATAAGAGACAG-3') and the 3'overhang adapter sequence (5'-GTCTCGTGGGCTCGGAGATGTGTATAAGAGACAG-3') for subsequent Illumina NGS amplicon sequencing, which was performed at the Genome Sequencing Facility (GSF) at The University of Texas Health Science Center at San Antonio (UTHSCSA). 2 million individual reads were generated for each sample. Subsequently, the relative abundances of all 93 sgRNA target sequences were calculated and represented as counts per million reads (CPM). Log-transformed values were used for presentation.

\section{Apoptosis assay}

To determine the non-apoptotic cell population 72 hours post-treatment with miR-34a mimic, $1 \mathrm{~mL}$ of the original cell growth medium was transferred into a $15 \mathrm{~mL}$ conical tube. Cells were washed with $1 \mathrm{~mL}$ of PBS solution, which was also collected. Next, cells were trypsinized using $150 \mu \mathrm{L}$ of trypsin-EDTA for 5 minutes at $37^{\circ} \mathrm{C}$. Subsequently, the trypsin-EDTA was neutralized using $2 \mathrm{~mL}$ of the original cell growth medium/PBS washing solution mixture. The cells were harvested by centrifugation at 1,000 rpm for 5 minutes. The cell pellet was then re-suspended in $1 \mathrm{~mL}$ PBS solution, then subjected to centrifugation at 1,000 rpm for 5 minutes. Apoptosis was quantified using the Dead Cell Apoptosis Kit with Annexin V Alexa Fluor ${ }^{\text {TM }} 488$ \& Propidium Iodide (PI) (Invitrogen, catalog \# V13241), following manufacturer's instructions. Briefly, the harvested cell pellets were re-suspended in $100 \mu \mathrm{L}$ of $1 \mathrm{X}$ annexin-binding buffer before being stained with $1 \mu \mathrm{L}$ propidium iodide $(100 \mu \mathrm{g} / \mu \mathrm{L})$ and $5 \mu \mathrm{L}$ of stock annexin $\mathrm{V}$, Alexa Fluor ${ }^{\mathrm{TM}} 488$ conjugate for 15 minutes in the dark. Stained cells were then diluted with $400 \mu \mathrm{L}$ of $1 \mathrm{X}$ annexin-binding buffer before subjected to flow cytometry. 
Excitation/emission wavelengths for the annexin V, Alexa Fluor ${ }^{\mathrm{TM}} 488$ conjugate are $495 / 519$ nm; for propidium iodide they are $533 / 617 \mathrm{~nm}$.

\section{Cell viability assay}

Approximately 150,000 of the HEK293, Flp-In-293, FLP-EDIT1 and FLP-EDIT2 cells were seeded into 6-well plates in $2 \mathrm{~mL}$ of complete medium. FLP-EDIT1 and FLP-EDIT2 cells were maintained with $0.5 \mu \mathrm{g} / \mathrm{mL}$ puromycin. All cells were treated with $100 \mu \mathrm{g} / \mathrm{mL}$ zeocin. For each cell type, 6 wells were included so that one of the wells could be harvested and counted on each day (from day 1 to day 6 after seeding). Three independent experiments were performed. For live cell counting, the cell suspension was mixed with $0.4 \%$ Trypan Blue solution (Invitrogen, catalog number: 15250) at a 1:1 ratio (volume:volume). Unstained, live cells were then counted using a hemocytometer (Hausser Scientific, catalog number: UX-79001-00) under a light microscope.

\section{Quantitative reverse transcription-PCR (qRT-PCR)}

For measurement of BCL2 mRNA levels, total RNA was extracted using the RNeasy Mini Kit (Qiagen, \#74104) 48 hours post-transfection. First strand synthesis was performed using the QuantiTect Reverse Transcription Kit (Qiagen, \#205311). Quantitative PCR was performed using the KAPA SYBR FAST Universal qPCR Kit (KAPABiosystems, \#KK4601), with GAPDH levels used for normalization. Quantitative analysis was performed using the $2^{-\Delta \Delta C t}$ method. Fold-change values are reported as mean with standard deviation. Primers used for BCL2 were (P4) 5'CATGCTGGGGCCGTACAG-3' and (P5) 5'-GAACCGGCACCTGCACAC-3'. Primers used for GAPDH were (P6) 5'AATCCCATCACCATCTTCCA-3' and (P7) 5'-TGGACTCCACGACGTACTCA-3'.

\section{General cloning protocol}

Q5 High-Fidelity 2X Master Mix (New England Biolabs, catalog number: M0492) was used for all polymerase chain reactions (PCR) according to the manufacturer's protocol. All oligonucleotides were ordered from SigmaAldrich and are listed in Supplementary Table 5. Plasmids were constructed using PCR amplification, restriction digest (all restriction enzymes were ordered from New England Biolabs), and ligation with T4 DNA ligase (New England Biolabs, catalog number: M0202S). Gel purification and PCR purification were performed 
with QIAquick Gel Extraction (catalog number: 28704) and PCR Purification kits (catalog number: 28104)

(Qiagen). Transformations were performed using NEB 5-alpha electrocompetent Escherichia Coli (New England Biolabs, catalog number: C2987P). Minipreps were performed using QIAprep Spin Miniprep kit (Qiagen, catalog number: 27104). The final plasmids were confirmed by both restriction enzyme digestion and direct Sanger sequencing.

\section{DNA Constructs}

U6-BCL2/sgRNA-PEF1: A pair of oligonucleotides (Forward: 5'-CACCGAATCAGCTATTTACTGCCAA-3', Reverse: 5'-AAACTTGGCAGTAAATAGCTGATTC-3') were annealed and cloned into the Esp3I-treated lentiCRISPRv2 plasmid. For more details, refer to Methods: Preparation of the CRISPR plasmid library.

\section{U6-zeocin resistance gene/sgRNA1-PEF1 and U6-zeocin resistance gene/sgRNA2-PEF1: The zeocin} resistance gene/sgRNA1 and zeocin resistance gene/sgRNA2 sequences were prepared from two rounds of PCR: first, the fragments were PCR amplified from U6-BCL2/sgRNA-PEF1 using primers P12 and P13 or P14; second, the final fragments were PCR amplified from the first round PCR products using primers P12 and P15. Subsequently, the PCR products were cloned into the U6-BCL2/sgRNA-PEF1 plasmid using KpnI and EcoRI sites.

\section{Cell culture and transient transfection}

HEK293 cells were acquired from American Type Culture Collection (ATCC, catalog number: CRL-1573). HCT116 wild-type and HCT 116 p53/-- cells were gifts from Dr. Michael A. White (University of Texas Southwestern Medical Center). Flp-In-293 cells were purchased from ThermoFisher Scientific (catalog number: R75007). All cell lines were maintained at $37^{\circ} \mathrm{C}, 100 \%$ humidity and $5 \% \mathrm{CO}_{2}$. Cells were grown in Dulbecco's modified Eagle's medium (DMEM, Invitrogen, catalog number: 11965-1181) supplemented with 10\% Fetal Bovine Serum (FBS, Invitrogen, catalog number: 26140), 0.1 mM MEM non-essential amino acids (Invitrogen, catalog number: $11140-050$ ), and 0.045 units/mL of Penicillin and 0.045 units/mL of streptomycin (PenicillinStreptomycin liquid, Invitrogen, catalog number: 15140). In addition, $100 \mu \mathrm{g} / \mathrm{mL}$ zeocin (ThermoFisher 
Scientific, catalog number: R25001) was used for maintaining the Flp-In-293 cells. To pass the cells, adherent cultures were first washed with PBS (Dulbecco's Phosphate Buffered Saline, Mediatech, catalog number: 21030-CM), then trypsinized with Trypsin-EDTA (0.25\% Trypsin with EDTAX4Na, Invitrogen, catalog number: 25200) and finally diluted in fresh medium. For transient transfection of miRNA mimics, $200 \mu \mathrm{L}$ of DMEM was mixed with $25 \mathrm{nM}$ (final concentration) of miR-34a mimic (Qiagen, catalog number: MSY0000255) or miR-cel-67 mimic (Dharmacon, catalog number: CN-001000-01), in addition to $2 \mu \mathrm{L}$ of RNAiMAX (Invitrogen, catalog number: 13778030). The mixture was added to each well of 12-well culture treated plastic plates (Greiner Bio-One, catalog number: 665180) and incubated at room temperature for 20 minutes. Adherent cell cultures were washed with PBS, then trypsinized with Trypsin-EDTA and finally diluted in fresh medium to the cell density of 200,000 cells $/ 800 \mu \mathrm{L}$ medium. $800 \mu \mathrm{L}$ of the diluted cell suspension was then added to the well containing the miRNA-RNAiMAX complex. 


\section{Acknowledgements}

This work was funded by the US National Science Foundation (NSF) CAREER grant 1351354, NSF 1361355, and the University of Texas at Dallas. We thank S. Lawson for technical support.

\section{Author contributions}

Y.L. and L.B. designed the experiments. Y.L., C.N., and D.W. performed the experiments. Y.L., C.N., A.P, and L. B. analyzed the data. Y.L., C.N. A.P, and L.B. wrote the paper. L.B. supervised the project.

\section{Competing financial interests}

The authors declare no competing financial interests.

\section{Additional information}

Supplementary information is available in the online version of the paper. Correspondence and requests for materials should be addressed to L.B. (bleris@utdallas.edu). 
bioRxiv preprint doi: https://doi.org/10.1101/265710; this version posted February 16, 2018. The copyright holder for this preprint (which was not certified by peer review) is the author/funder, who has granted bioRxiv a license to display the preprint in perpetuity. It is made available under aCC-BY 4.0 International license.

\section{Figures}
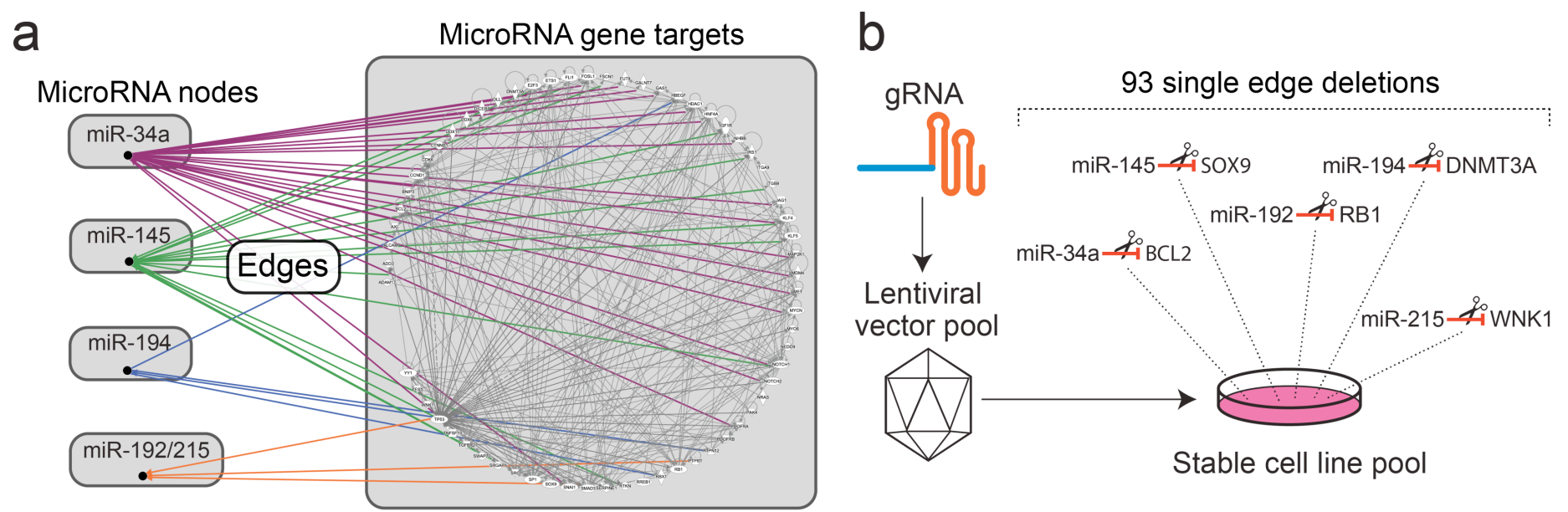

Figure 1. p53-miRNA network and CRISPR-based edge screens. (a) Complexity of the p53-miRNA network with nodes comprising the indicated miRNAs and their 71 target genes and edges based on experimentally verified and high-confidence predicted direct interactions, derived using Qiagen Ingenuity Pathway Analysis. (b) CRISPR-based lentiviral libraries were prepared using the lentiCRISPRv2 system. The stably integrated CRISPR sgRNA constructs were recovered by PCR and the sgRNA targets were identified using NGS. 
a

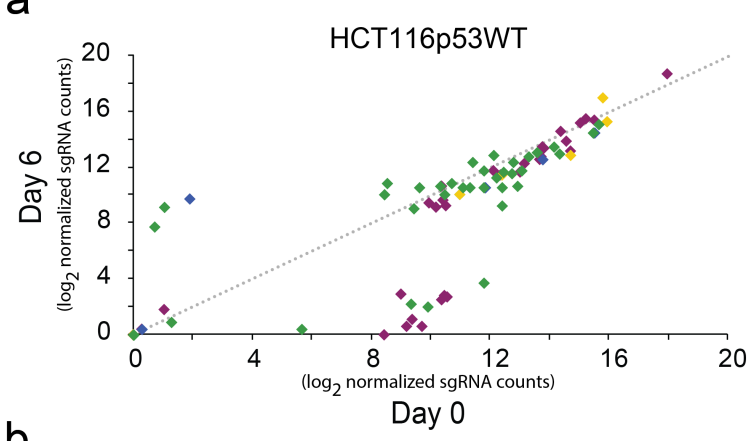

b

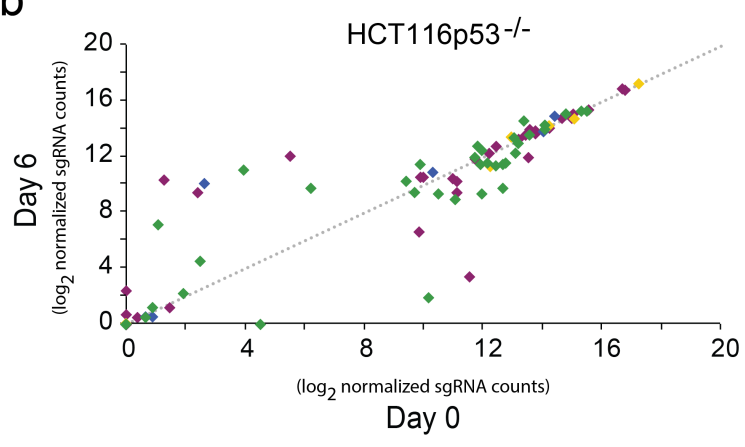

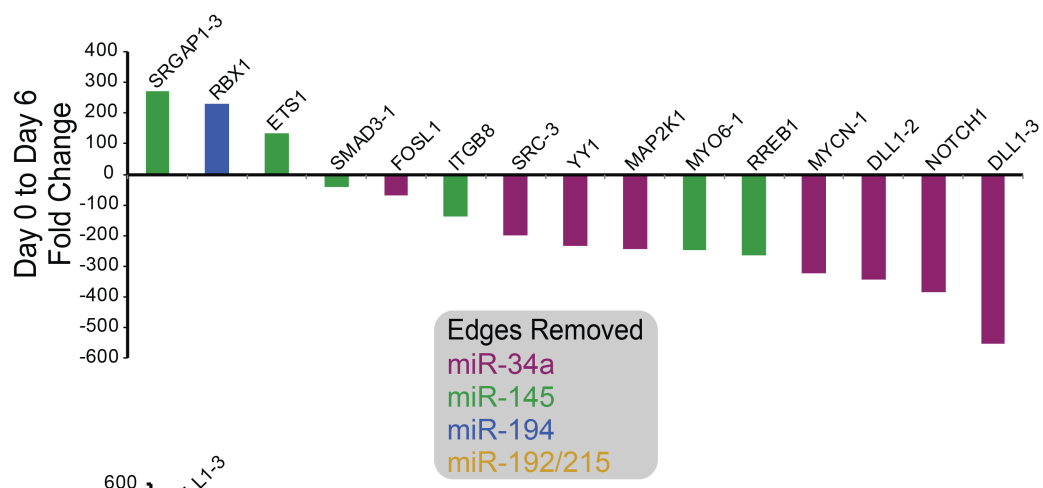

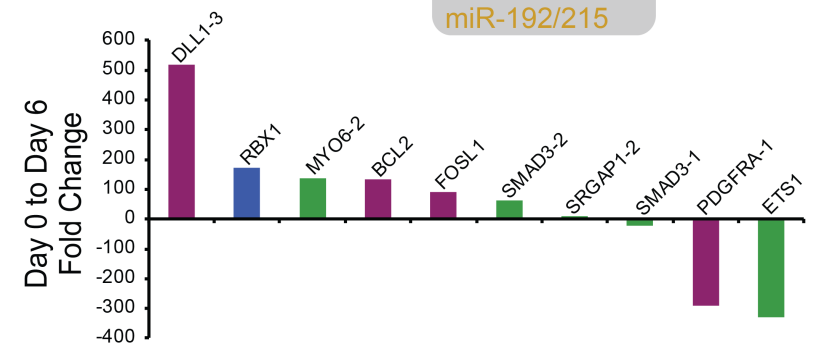

Figure 2. High-throughput editing of edges with CRISPR libraries in (a) HCT116p53WT (LIB-WT) cells and (b) HCT116p53 ${ }^{-/-}\left(\right.$LIB-p53 $\left.^{-/}\right)$. The sgRNA targets showing the highest folder changes (> 10) after 6 days treatment of $25 \mathrm{nM}$ with miR-34a mimic are shown, with positive values indicating enrichment and negative values indicating depletion. 
bioRxiv preprint doi: https://doi.org/10.1101/265710; this version posted February 16, 2018. The copyright holder for this preprint (which was not certified by peer review) is the author/funder, who has granted bioRxiv a license to display the preprint in perpetuity. It is made available under aCC-BY 4.0 International license.

a

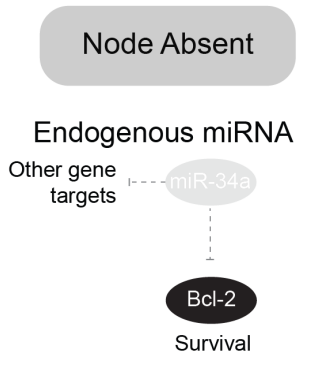

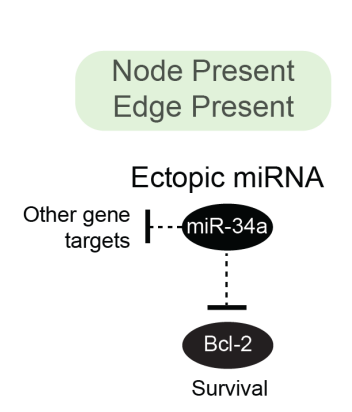
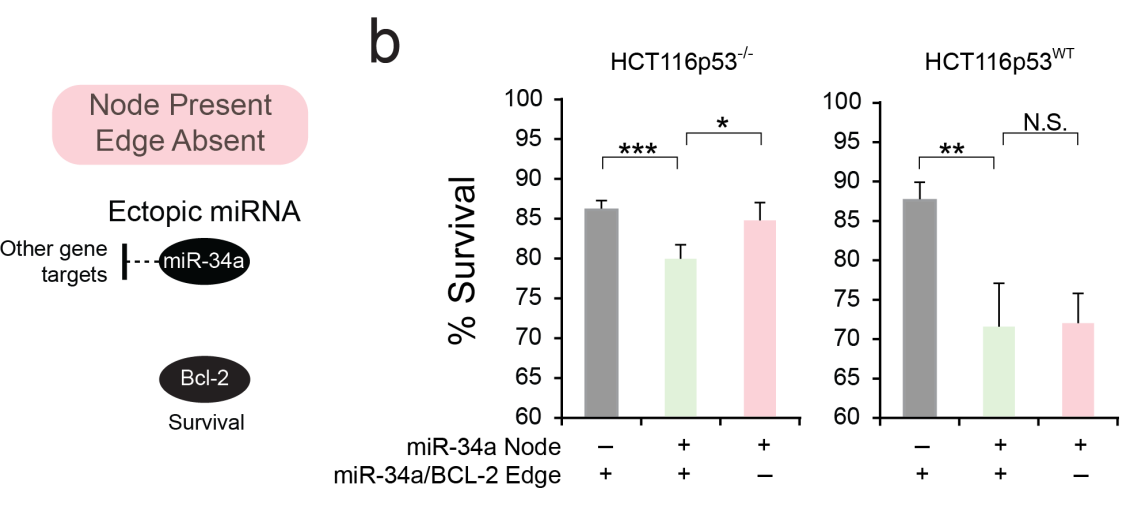

Figure 3. Survival analysis after ectopic miR-34a delivery using node vs. edge approaches. (a) Schematic illustration of node vs. edge analysis. Ectopic miR-34a represents a network node and the miR-34a/Bcl-2 interaction represents a network edge. (b) The node-based approach shows that addition of the miR-34a node induces apoptosis in both p53 WT and deficient cells. In contrast, the edge-based approach reveals that introduction of the miR-34a/Bcl-2 edge induces apoptosis only in the p53-deficient cells and not in p53-WT cells. 


\section{References}

1. A. B. DeLeo et al., Detection of a transformation-related antigen in chemically induced sarcomas and other transformed cells of the mouse. Proc. Natl. Acad. Sci. U. S. A. 76, 2420-4 (1979).

2. K. H. Vousden, C. Prives, Blinded by the Light: The Growing Complexity of p53. Cell. 137, 413-431 (2009).

3. A. J. Levine, p53, the cellular gatekeeper for growth and division. Cell. 88, 323-31 (1997).

4. E. Batchelor, A. Loewer, G. Lahav, The ups and downs of p53: understanding protein dynamics in single cells. Nat. Rev. Cancer. 9, 371-377 (2009).

5. L. He et al., A microRNA component of the p53 tumour suppressor network. Nature. 447, 1130-1134 (2007).

6. V. Tarasov et al., Differential Regulation of microRNAs by p53 Revealed by Massively Parallel Sequencing: miR-34a is a p53 Target That Induces Apoptosis and G(1)-arrest. Cell Cycle. 6 (2007) (available at http://view.ncbi.nlm.nih.gov/pubmed/17554199).

7. D. P. Bartel, MicroRNAs: Target Recognition and Regulatory Functions. Cell. 136, 215-233 (2009).

8. W. Filipowicz, S. N. Bhattacharyya, N. Sonenberg, Mechanisms of post-transcriptional regulation by microRNAs: are the answers in sight? Nat Rev Genet. 2008, 102-114 (2008).

9. R. Moore, H. K. Ooi, T. Kang, L. Bleris, L. Ma, microRNA-192-mediated positive feedback loop controls the robustness of stress-induced p53 oscillations in breast cancer cells. PLoS Comput. Biol. 11, e1004653 (2015)

10. J. F. Abelson et al., Sequence variants in SLITRK1 are associated with Tourette's syndrome. Science. 310, 317-320 (2005).

11. A. Chatzikyriakidou, P. V Voulgari, I. Georgiou, A. A. Drosos, A polymorphism in the 3'-UTR of interleukin-1 receptor-associated kinase (IRAK1), a target gene of miR-146a, is associated with 
rheumatoid arthritis susceptibility. Joint. Bone. Spine. 77, 411-413 (2010).

12. C. R. Consiglio et al., Association of the HLA-G gene $+3142 \mathrm{C}>\mathrm{G}$ polymorphism with systemic lupus erythematosus. Tissue Antigens. 77, 540-545 (2011).

13. L.-S. Wu et al., A miRNA-492 binding-site polymorphism in BSG (basigin) confers risk to psoriasis in central south Chinese population. Hum. Genet. 130, 749-757 (2011).

14. P. Brest et al., A synonymous variant in IRGM alters a binding site for miR-196 and causes deregulation of IRGM-dependent xenophagy in Crohn's disease. Nat. Genet. 43, 242-245 (2011).

15. S. Kulkarni et al., Genetic interplay between HLA-C and MIR148A in HIV control and Crohn disease. Proc. Natl. Acad. Sci. U. S. A. 110, 20705-20710 (2013).

16. G. Wang et al., Variation in the miRNA-433 binding site of FGF20 confers risk for Parkinson disease by overexpression of alpha-synuclein. Am. J. Hum. Genet. 82, 283-289 (2008).

17. P. Sethupathy et al., Human microRNA-155 on chromosome 21 differentially interacts with its polymorphic target in the AGTR1 3' untranslated region: a mechanism for functional single-nucleotide polymorphisms related to phenotypes. Am. J. Hum. Genet. 81, 405-413 (2007).

18. G. Hanin et al., Competing targets of microRNA-608 affect anxiety and hypertension. Hum. Mol. Genet. 23, 4569-4580 (2014).

19. K. Lv et al., Allele-specific targeting of hsa-miR-657 to human IGF2R creates a potential mechanism underlying the association of ACAA-insertion/deletion polymorphism with type 2 diabetes. Biochem. Biophys. Res. Commun. 374, 101-105 (2008).

20. K. Richardson et al., The PLIN4 variant rs8887 modulates obesity related phenotypes in humans through creation of a novel miR-522 seed site. PLoS One. 6, e17944 (2011).

21. B. M. Ryan et al., Identification of a functional SNP in the 3'UTR of CXCR2 that is associated with reduced risk of lung cancer. Cancer Res. 75, 566-575 (2015). 
22. L. J. Chin et al., A SNP in a let-7 microRNA Complementary Site in the KRAS 3' Untranslated Region Increases Non-Small Cell Lung Cancer Risk. Cancer Res. 68 (2008).

23. Y. Zu et al., Genetic variation in a miR-335 binding site in BIRC5 alters susceptibility to lung cancer in Chinese Han populations. Biochem. Biophys. Res. Commun. 430, 529-534 (2013).

24. F. Xiong et al., Genetic variation in an miRNA-1827 binding site in MYCL1 alters susceptibility to small-cell lung cancer. Cancer Res. 71, 5175-5181 (2011).

25. X. Pu et al., MicroRNA-related genetic variants associated with clinical outcomes in early-stage nonsmall cell lung cancer patients. Cancer Res. 73, 1867-1875 (2013).

26. N. Okada et al., A positive feedback between p53 and miR-34 miRNAs mediates tumor suppression. Genes Dev. 28, 438-50 (2014).

27. P. Mali et al., RNA-guided human genome engineering via Cas9. Science. 339, 823-826 (2013).

28. Z. Rong, S. Zhu, Y. Xu, X. Fu, Homologous recombination in human embryonic stem cells using CRISPR/Cas9 nickase and a long DNA donor template. Protein Cell. 5, 258-60 (2014).

29. J.-F. Li et al., Multiplex and homologous recombination-mediated genome editing in Arabidopsis and Nicotiana benthamiana using guide RNA and Cas9. Nat. Biotechnol. 31, 688-91 (2013).

30. T. Wang, J. J. Wei, D. M. Sabatini, E. S. Lander, Genetic Screens in Human Cells Using the CRISPRCas9 System. Science (80-. ). 343, 80-84 (2014).

31. S. Chen et al., Genome-wide CRISPR Screen in a Mouse Model of Tumor Growth and Metastasis. Cell. 160, 1246-1260 (2015).

32. S. Zhu et al., Genome-scale deletion screening of human long non-coding RNAs using a paired-guide RNA CRISPR-Cas9 library. Nat. Biotechnol. 34, 1279-1286 (2016).

33. F. Navarro, J. Lieberman, miR-34 and p53: New Insights into a Complex Functional Relationship. PLoS One. 10, e0132767 (2015). 
34. P. Sundaram et al., p53-Responsive miR-194 Inhibits Thrombospondin-1 and Promotes Angiogenesis in Colon Cancers. Cancer Res. 71, 7490-7501 (2011).

35. Z. Feng, C. Zhang, R. Wu, W. Hu, Tumor suppressor p53 meets microRNAs. J. Mol. Cell Biol. 3, 44-50 (2011).

36. C.-H. Chou et al., miRTarBase 2016: updates to the experimentally validated miRNA-target interactions database. Nucleic Acids Res. 44, D239-D247 (2016).

37. V. Agarwal, G. W. Bell, J.-W. Nam, D. P. Bartel, Predicting effective microRNA target sites in mammalian mRNAs. Elife. 4, e05005 (2015).

38. D. P. Bartel, MicroRNAs: target recognition and regulatory functions. Cell. 136, 215-33 (2009).

39. N. E. Sanjana, O. Shalem, F. Zhang, Improved vectors and genome-wide libraries for CRISPR screening. Nat. Methods. 11, 783-784 (2014).

40. O. Shalem et al., Genome-Scale CRISPR-Cas9 Knockout Screening in Human Cells. Science (80-. ). 343, 84-87 (2014).

41. T.-C. Chang et al., "Transactivation of miR-34a by p53 Broadly Influences Gene Expression and Promotes Apoptosis" (2007), , doi:10.1016/j.molcel.2007.05.010.

42. A. G. Bader, miR-34 - a microRNA replacement therapy is headed to the clinic. Front. Genet. 3, 120 (2012).

43. L. Jia, Y. Sun, RBX1/ROC1-SCF E3 ubiquitin ligase is required for mouse embryogenesis and cancer cell survival. Cell Div. 4, 16 (2009).

44. S. Cory, J. M. Adams, The Bcl-2 family: regulators of the cellular life-or-death switch. Nat Rev Cancer. 2, 647-656 (2002).

45. T. Kang et al., Discriminating direct and indirect connectivities in biological networks. Proc. Natl. Acad. Sci. U. S. A. 112, 201507168 (2015). 
bioRxiv preprint doi: https://doi.org/10.1101/265710; this version posted February 16,2018 . The copyright holder for this preprint (which was not certified by peer review) is the author/funder, who has granted bioRxiv a license to display the preprint in perpetuity. It is made available under ACC-BY 4.0 International license. 